\title{
EFEITOS DA UTILIZAÇÃO DE UM APLICATIVO DE CELULAR EM PARTICIPANTES DE UM PROGRAMA ESPECIALIZADO EM CESSAÇÃO TABAGISTICA: ESTUDO CLÍNICO RANDOMIZADO
}

\author{
Rhuan Gustavo Duran Miron ${ }^{1}$, Isabela Martins Peruque ${ }^{1}$, Bruna De Souza Goulart ${ }^{1}$, Ester Teixeira Santos ${ }^{1}$, \\ Mariane Magro Marcelino Soares ${ }^{1}$, Tainá Oliveira Lopes ${ }^{1}$, Flávio Danilo Mungo Pissulin ${ }^{1}$, Robson Augusto \\ Siscoutto ${ }^{1}$, Juliana Souza Uzeloto ${ }^{2}$, Ana Paula Coelho Figueira Freire ${ }^{1}$ \\ ${ }^{1}$ Universidade do Oeste Paulista - UNOESTE, Presidente Prudente, SP. ${ }^{2}$ Universidade Estadual Paulista Júlio de \\ Mesquita Filho - UNESP, Presidente Prudente, SP. E-mail: rhuan.miron@hotmail.com
}

\section{RESUMO}

O objetivo do estudo foi analisar os efeitos da utilização de um aplicativo de celular na cessação tabagística e na satisfação de participantes de um programa especializado. Estudo clínico randomizado do qual participaram do estudo 25 indivíduos de um programa de cessação tabagística especializado por um período de 12 semanas. Não foram detectadas diferenças significativas no índice de cessação ( $p=0.1317)$ e recaídas $(p=1.000)$. Na intensidade da síndrome de abstinência e a variação absoluta (delta) identificou redução significativa apenas para o $\operatorname{GApp}(p=0.0165)$, na comparação do delta entre grupos não foram identificadas diferenças estatisticamente significativas $(p=0.1066)$. No questionário de satisfação geral os participantes relataram facilidade nos aspectos analisados. Não foram encontradas diferenças significativas pelos GApp e GC ( $p>0,05)$. Conclui-se que o aplicativo de celular não influenciou na cessação do tabagismo, na sindrome de abstinência e na satisfação de tabagistas ao ser usado em participantes em um programa especializado.

Palavras-chave: tabagismo, cessação tabágica, aplicativos móveis,síndrome de abstinência a substâncias, satisfação pessoal.

\section{EFFECTS OF USING A CELL PHONE APP ON PARTICIPANTS OF A SPECIALIZED PROGRAM ON SMOKING CESSATION: RANDOMIZED CLINICAL STUDY}

\begin{abstract}
The goal of the study was to analyze the effects of using a cell cessation application and the satisfaction of participants in a specialized program. Randomized clinical study in which participates in study 25 , a specialized smoking cessation program for a period of 12 weeks. No significant differences were detected in the cessation rate $(p=0.1317)$ and returned $(p=1,000)$. The intensity of the abstinence syndrome and absolute variation (delta) identified a reduced reduction only for the GApp ( $p=0.0165)$, when comparing the delta between unidentified groups with statistically significant differences $(p=0.1066)$. In the general satisfaction questionnaire, the participants reported the ease in the aspects analyzed. There were no significant differences for GApp and GC ( $p>0.05$ ). Conclude if the cell phone application did not influence smoking cessation, withdrawal syndrome and smokers' satisfaction when used in participants in a specialized program.
\end{abstract}

Keywords: tobacco use disorder, tobacco use cessation, mobile applications, substance withdrawal syndrome, personal satisfaction. 


\section{INTRODUÇÃO}

Em todo o mundo a prevalência de fumantes é de 1,1 bilhão de pessoas, no Brasil existem cerca de 27,9 milhões de fumantes, consumindo 110 bilhões de cigarros por ano do qual ocorrem $200 \mathrm{mil}$ óbitos anualmente relacionado ao tabaco ${ }^{1,2}$. O tabagismo se tornou um grave problema de saúde pública, sendo considerado um fator de risco para diversas doenças ${ }^{3}$.

Estudos demonstraram que $80 \%$ dos indivíduos tabagistas apresentam o desejo de parar de fumar, porém apenas 3\% conseguem sem nenhum tipo de auxílio. Frente a este dado, destaca-se a importância de programas especializados para cessação tabagística ${ }^{4}$. 0 tabagismo pode ser tratado com eficácia por terapias com abordagem na dependência comportamental, química e psíquica ${ }^{5,6}$.

Dentre estes programas de intervenção podem-se citar programas estruturados que utilizam terapia cognitivo-comportamental (TCC) que podem ser constituídos de terapias em grupo para o abandono do cigarro ${ }^{7}$. Neste tipo de abordagem os principais objetivos incluem a detecção de situações de risco de recaída e o desenvolvimento de estratégias para manutenção da abstinência, do qual o acompanhamento é fundamental para que se obtenha sucesso com a abordagem ${ }^{8}$.

Em relação à eficácia, estudos apontaram que as terapias cognitivo-comportamentais são eficazes mesmo sem o uso de medicamentos; já os medicamentos utilizados de maneira isolada proporcionam menores taxas de sucesso ${ }^{9}$. Assim, a terapia farmacológica deve ser utilizada como complemento aos programas, o qual representa um recurso valioso, ajudando a amenizar os sintomas da síndrome de abstinência ${ }^{10,11}$.

Apesar de bons resultados obtidos nos programas presenciais de tratamento ao tabagismo a taxa de abandono e desistência ainda pode ser considerada como fator limitante para o tratamento ${ }^{12,13}$. Assim observa-se que o tratamento do tabagismo ainda necessita de certas adequações para tornar-se cada vez mais efetivo ${ }^{14}$.

O rápido crescimento do número de smartphones e computadores com acesso à internet propiciou a criação de diversos aplicativos para complementar o tratamento de saúde $^{15}$. Aplicativos para smartphones também vêm sendo desenvolvidos como estratégias para cessação do tabagismo, pois podem oferecer educação e aconselhamento para diversos tipos de usuários 24 horas por dia. Além disso, as estratégias são atualizadas rapidamente e facilitam o acesso ás informações de tratamento ao usuário ${ }^{16}$.

Entretanto, mesmo com o crescimento ao acesso de tecnologia no país, as informações disponíveis na internet sobre o tabagismo apresentam problemas de qualidade, além de apresentarem uma cobertura limitada dos tópicos recomendados pela diretriz de tratamento ao tabagismo, principalmente os aplicativos em português ${ }^{16,17}$. Além disso, os aplicativos existentes geralmente não estão vinculados á um programa presencial estruturado ${ }^{18-20}$.

Dessa forma o tabagista não tem o apoio presencial de uma equipe especializada, apenas o suporte tecnológico, o que pode ser menos efetivo, tornando-se necessário o desenvolvimento de propostas que associem todos os benefícios do apoio presencial dos programas estruturados para cessação com as vantagens ofertadas pelas novas tecnologias disponíveis nos aplicativos de celular. Acreditase que estas medidas podem inovar e agregar potencial ao processo de cessação do tabagismo, pois o acesso à informações precisas, rápidas e de fácil acesso podem reduzir recaídas e desistências dos pacientes, ainda mais quando associadas ao suporte presencial de um programa estruturado.

Assim, o objetivo do estudo foi analisar os efeitos da utilização de um aplicativo de celular associado ao tratamento presencial na taxa de cessação tabagística, intensidade da síndrome de abstinência e satisfação de participantes de um programa especializado.

\section{MÉTODOS \\ ASPECTOS ÉTICOS}

Trata-se de um estudo clínico randomizado, submetido e aprovado pelo Comitê de Ética em Pesquisa (CAAE. 03653218.5.0000.5515; número do parecer 3.124.368) e Registro Brasileiro de ensaios clínicos (ReBEC) RBR-775D8M.

\section{SELEÇÃO DA AMOSTRA}

Os participantes inseridos no estudo foram instruídos em relação a todos os procedimentos executados e assinaram o termo de consentimento livre e esclarecido 
concordando em participarem do presente estudo. Foram avaliados 70 tabagistas por amostra de conveniência, participantes de um programa de cessação tabagística especializado. Os mesmos foram incluídos após buscarem voluntariamente $\mathrm{o}$ programa em uma Universidade do Oeste Paulista e manifestaram o desejo em parar de fumar. Os critérios de inclusão foram participantes com mais de 18 anos, tabagistas há mais de um ano, sem comorbidades psiquiátricas severas como esquizofrenia ou bipolaridade previamente diagnosticada e que possuíam smartphone compatível com o aplicativo utilizado no estudo. Foram excluídos participantes analfabetos, que abandonaram o tratamento durante a execução do estudo e aqueles que possuíam smartphone IOS sendo incompatível com o aplicativo.

Desenho Experimental

Inicialmente os participantes realizaram uma avaliação que contemplou dados gerais, comorbidades e histórico de tabagismo. Também foi aplicada a escala para avaliação da intensidade de sintomas de abstinência à nicotina ${ }^{21}$. Em seguida os participantes foram incluídos em um programa especializado para cessação do tabagismo que utiliza abordagem intensiva no auxílio para abandono do tabaco.

\section{SÍNDROME DA ABSTINÊNCIA DO TABAGISMO - QUESTIONÁRIO WSWS}

Foi realizada ao início do estudo uma avaliação inicial por meio de entrevista que englobou dados sociodemográficos e histórico de tabagismo. Na avaliação inicial e após 5 semanas, foi aplicada a Wisconsin Smoking Withdrawal Scale a qual possui 28 questões, divididas em sete domínios, com pontuação que varia de 0 (zero) a 4 (quatro) pontos para cada uma e avalia os principais sintomas da síndrome de abstinência à nicotina, num somatório máximo de 112 pontos. A dependência é maior quanto maior a pontuação obtida na escala ${ }^{21}$.

\section{PROGRAMA ESPECIALIZADO DE CESSAÇÃO DO TABAGISMO}

O programa presencial consistiu em um tratamento especializado com abordagem intensiva (uma vez na semana com duração de uma hora e trinta minutos) com um total de 3 meses, sendo o monitoramento de cessação de 5 semanas. As ações foram desenvolvidas por uma equipe interdisciplinar que incluiu as áreas de Fisioterapia, Nutrição, Medicina, Fonoaudiologia,
Estética e Odontologia. As atividades propostas no programa seguiram recomendações do Ministério da Saúde e Instituto Nacional do Câncer e baseou-se na associação da Terapia Cognitivo Comportamental (TCC) com terapia farmacológica especializada. Todos foram avaliados pela equipe médica para determinação da necessidade de terapia farmacológica. A prescrição englobou o uso da terapia de reposição de nicotina por meio de adesivos transdérmicos associado com cloridrato de bupropiona $(150 \mathrm{mg})^{20}$.

A primeira etapa do tratamento consistiu na fase educacional que incluiu palestras educativas como benefícios da cessação do fumo, ganho de peso, estratégias no combate da fissura, importância da atividade física para o tratamento e mudança de hábitos de vida.

$\mathrm{Na}$ segunda etapa do tratamento após a primeira tentativa de cessação definitiva, deu-se início a fase de intervenção, na qual os participantes tiveram espaço para expor relatos individuais sobre sua experiência no abandono do tabaco, expor suas dificuldades, interagir com o grupo, e compartilhar desafios e conselhos. Foi realizada interação entre equipe e pacientes por meio da Terapia Cognitivo Comportamental (TCC) reforçando atitudes que favorecem a permanência da abstinência e também identifica e monitora situações de risco. Por fim foi realizada a etapa de manutenção, na qual o grupo recebeu o acompanhamento de outros profissionais (Odontologia, Estética, Nutrição e Fonoaudiologia).

No início do programa os participantes foram aleatorizados (randomização via Excel $^{\circledR}$ ) em dois grupos de intervenção: Grupo Aplicativo (GApp), que participaram de todas as etapas do programa de cessação presencialmente e adicionalmente utilizaram um aplicativo de celular (Smokerstop $\left.{ }^{\circledR}\right)$, com recursos e ferramentas extras como medidas complementares para cessação. O segundo grupo (Grupo Controle - GC) foi formado por indivíduos que participaram do programa de cessação, entretanto sem a utilização do aplicativo de celular. Após um período de 5 semanas de cessação foram analisados o índice de cessação tabagística, a intensidade dos sintomas de abstinência e o nível de satisfação dos participantes de ambos os grupos.

Após o dia da cessação definitiva (definida pelos terapeutas) foi acrescentado a aplicação de um questionário elaborado pelos 
pesquisadores realizado semanalmente que monitorou a situação de abstinência e ocorrência de lapsos ou recaídas por meio do auto relato. Sendo considerada a cessação tabagistica aqueles que estavam abstinentes na data da aplicação do questionário, mesmo que ocorreram recaídas durante este período.

\section{APLICATIVO MÓVEL - SMOKERSTOP}

Para os participantes alocados no GApp, foi ofertado além de todas as etapas do programa presencial, a associação de recursos adicionais por meio de um aplicativo de celular denominado Smokerstop, o qual utiliza abordagem informativa para auxílio no processo de cessação. $O$ aplicativo individualiza a tentativa de abandono, permitindo a definição de metas pessoais, visualiza a recuperação do corpo a partir do abandono do tabaco, ajuda com dicas para parar de fumar e lembra as vantagens de não fumar ${ }^{22}$.

\section{QUESTIONÁRIO DE SATISFAÇÃO}

Também foi aplicado um questionário de satisfação para ambos os grupos, avaliando a experiências dos indivíduos mediante a participação do programa de cessação do tabagismo. Este questionário foi desenvolvido baseado em estudos prévios e modificado pelos próprios pesquisadores, sendo sua pontuação com valores de 0 a 10 (0 sendo fácil, 5 relativamente fácil e 10 muito difícil). As seguintes nove questões foram aplicadas:

Foi difícil entrar em contato com a equipe? Foi difícil parar de fumar? Foi mais difícil que você pensava antes de participar do grupo? Você se sentiu inseguro durante o processo? Você pensou em desistir? Você se sente desmotivado para continuar sem fumar? Achou difícil seguir as estratégias aconselhadas? Achou difícil lembrar do horário da medicação? Achou difícil lembrar de trocar os adesivos de nicotina?

Para o GApp foram elaboradas questões específicas sobre o uso do aplicativo. Este questionário foi desenvolvido baseado em estudos prévios e modificado pelos próprios pesquisadores, sendo sua pontuação com valores de 0 a 10 (0 sendo pouco satisfeito, 5 relativamente satisfeito e 10 muito satisfeito. As seguintes cinco questões foram aplicadas:

Foi fácil manusear o Aplicativo? O App trouxe benefícios durante 0 tratamento? Achou interessante os conteúdos disponíveis do App? Achou necessário o auxílio do App durante seu tratamento? O App foi útil nos momentos de fissura?

\section{ANÁLISE DOS DADOS}

Para análise dos dados foi utilizado software estatístico Graph Pad Prism. Para análise de comparação entre o percentual de cessação tabagística entre os dois grupos foi aplicado teste de Qui-quadrado. As análises de comparação sobre os índices de satisfação e intensidade de abstinência foram analisados pelo Teste $T$ de Student não pareado ou Mann Whitney de acordo com a normalidade dos dados. $O$ nível de significância utilizado foi de $5 \%$.

\section{RESULTADOS}

Foram avaliados 70 participantes tabagistas de ambos os sexos, sendo que após seguimento finalizaram as análises 25 participantes sendo 15 do Grupo Controle e 10 do Grupo Aplicativo. (Figura 1) 


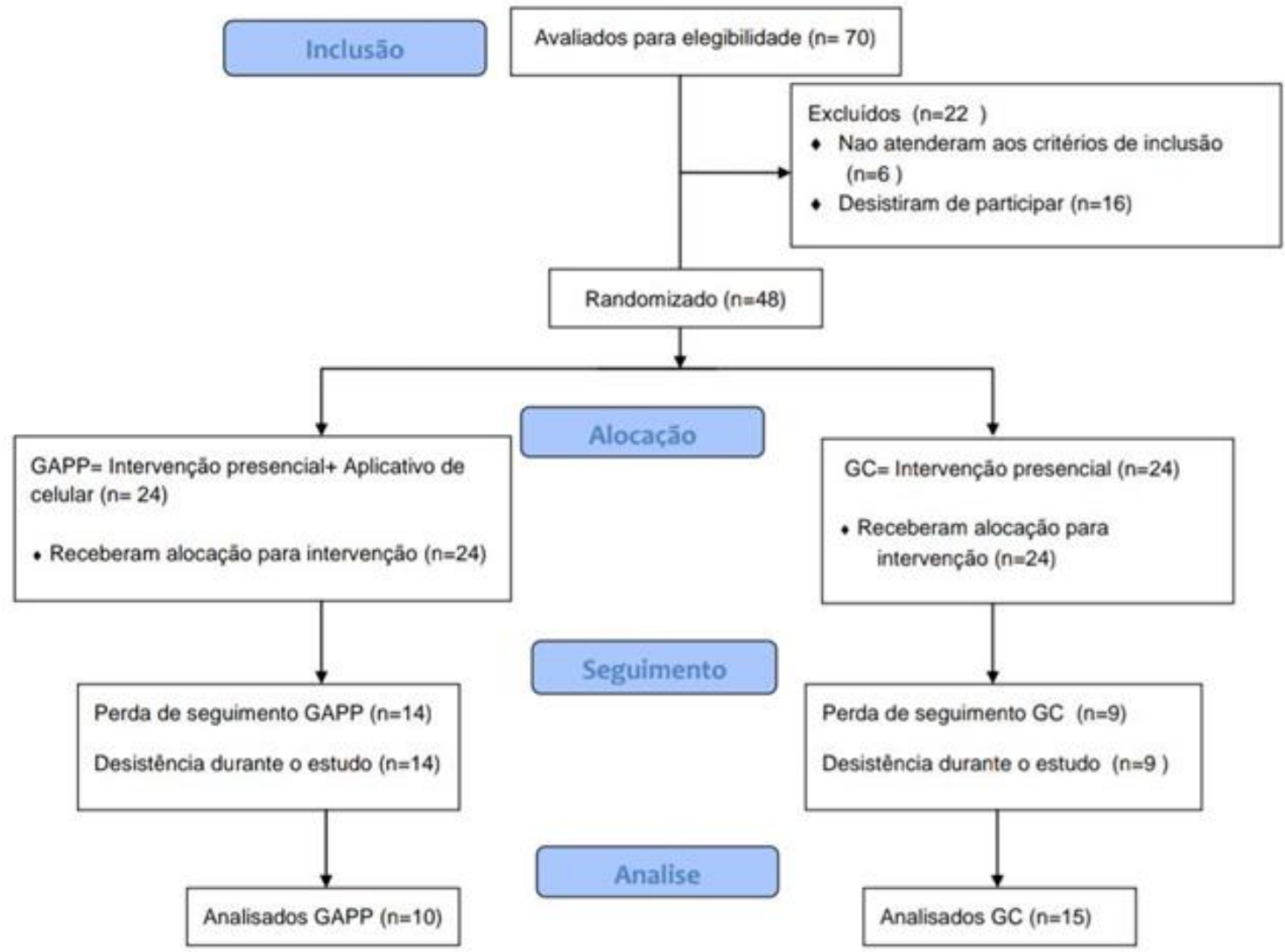

Figura 1. Fluxograma CONSORT de alocação, seguimento e análise dos participantes

Pode-se observar que nas características inicias e demográficas dos participantes o GC apresentou idade mais avançada e histórico de fumo maior quando comparado com o GApp.
Para o restante dos dados não foram detectadas diferenças significativas. (Tabela 1)

Tabela 1. Características demográficas e histórico de tabaco dos participantes do estudo.

\begin{tabular}{llll}
\hline $\begin{array}{l}\text { Dados Demográficos } \\
\text { Sexo } \mathrm{n}(\%)\end{array}$ & GApp & GC & Valor $\mathrm{p}$ \\
Feminino & & & \\
Masculino & $30 \%$ & $46 \%$ & \\
Idade & $70 \%$ & $54 \%$ & 0,4047 \\
Peso (Kg) & $39,30 \pm 12,86$ & $53,07 \pm 9,58$ & 0,0054 \\
Altura (cm) & $74,99 \pm 9,07$ & $76,99 \pm 14,31$ & 0,6998 \\
IMC (Kg/m2) & $1,72 \pm 0,11$ & $1,65 \pm 0,11$ & 0,1597 \\
Histórico tabagismo & $25,17 \pm 3,86$ & $28,01 \pm 4,10$ & 0,0969 \\
Anos de fumo & & & \\
Cigarros por dia & $23,60 \pm 13,42$ & $34,47 \pm 11,29$ & 0,0391 \\
Já tentou para de fumar(\%) & $22,30 \pm 11,86$ & $18,73 \pm 9,05$ & 0,4027 \\
Usou medicação (\%) & $80 \%$ & $66 \%$ & 0,4670 \\
Recaidas (\%) & $20 \%$ & $73 \%$ & 0,7022 \\
\hline \hline
\end{tabular}

Legenda: IMC: índice de massa corpórea.

Colloq Vitae 2020 set-dez; 12(3): 37-46.

Artigo Open Access sob uma licença CC BY-NC-ND (http://creativecommons.org/licenses/by-nc-nd/4.0/). 
Nos índices de cessação e de recaídas ao longo do período de acompanhamento, não foram identificadas diferenças significativas para esses índices, sendo $(p=0,1317)$ e $(p=1,000)$ respectivamente. (Figura 2 )
Índice Cessação

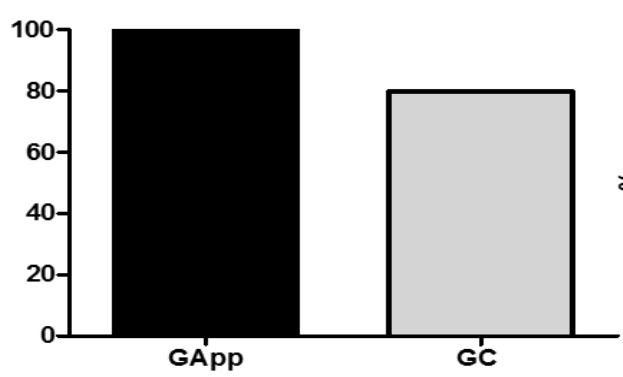

Índice Recaídas

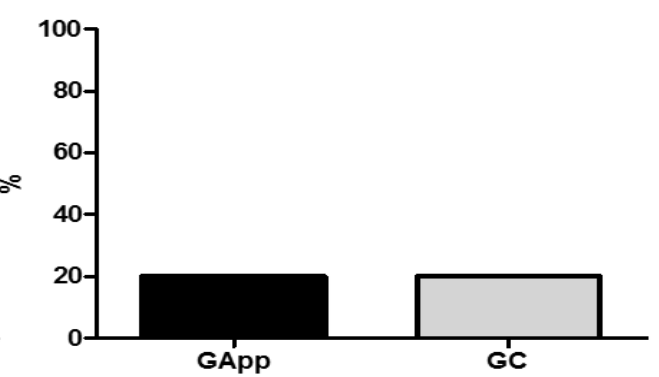

Figura 2. Índice percentual de cessação e recaídas dos grupos avaliados. Legenda: GApp: Grupo aplicativo, CG: Grupo controle.

Nos dados obtidos sobre a intensidade da síndrome de abstinência por meio do escore da Wisconsin Withdrawl Scale e também a variação absoluta (delta) antes e após o período de acompanhamento, observou-se que nas análises pareadas foi identificada redução significativa apenas para o GApp ( $p=0,0165)$. O GApp apresentou media do delta $-12,80 \pm 13,19$ e o GC $-1,93 \pm 17,34$ sendo a comparação dos deltas não significativas $(p=0,1066)$. (Figura 3 )
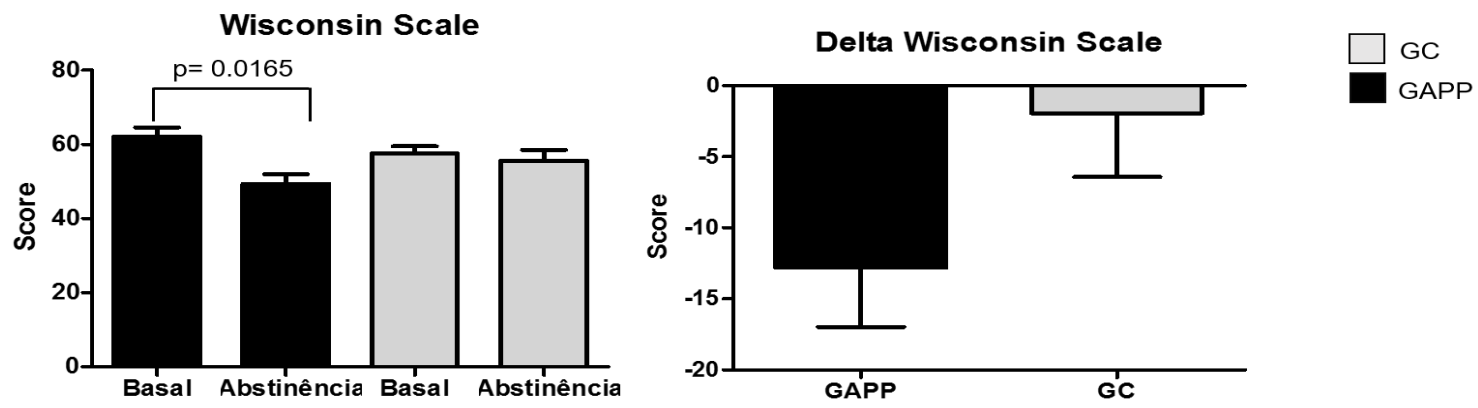

Figura 3. Comparação da avaliação de Wisconsin Withdrawl Scale antes e após a cessação e variação absoluta (delta) de ambos os grupos.

Nas análises descritivas de satisfação específicas para o GApp, observaram-se altos índices de satisfação (próximos a 10). Já para o questionário de satisfação geral, aplicado para ambos os grupos, os participantes relataram facilidade (dados mais próximos de 0) nos aspectos analisados. Além disso, não foram encontradas diferenças significativas nas pontuações atribuídas pelos GApp e GC $(p>0,05)$. (Tabela 2) 
Tabela 2. Questionário desenvolvido pelos próprios pesquisadores para avaliar índice de satisfação do uso do aplicativo e da intervenção presencial.

\section{Questionário de satisfação GAPP}

1. Foi fácil manusear o Aplicativo?

$9,40 \pm 1,57$

2. O App trouxe benefícios durante o tratamento?

$8,80 \pm 2,09$

3. Achou interessante os conteúdos disponíveis do App?

$9,30 \pm 1,63$

4. Achou necessário o auxílio do App durante seu tratamento?

$7,90 \pm 2,51$

5. O App foi útil nos momentos de fissura?

Questionário de satisfação para ambos os grupos

$8,00 \pm 2,40$

1. Foi difícil entrar em contato com a equipe?

\section{GAPP}

GC

Valor $p$

2. Foi difícil parar de fumar?

$0,50 \pm 1,58$

$0 \pm 0$

3. Foi mais difícil que você pensava antes de participar do grupo?

$5,20 \pm 3,96 \quad 5,60 \pm 3,97 \quad 0,7757$

4. Você se sentiu inseguro durante o processo?

$4,80 \pm 2,97 \quad 3,13 \pm 4,48 \quad 0,2180$

5. Você pensou em desistir?

$3,50 \pm 3,40 \quad 5,33 \pm 4,46 \quad 0,3566$

$3,30 \pm 2,98 \quad 1,33 \pm 3,51 \quad 0,0550$

6. Você se sente desmotivado para continuar sem fumar?

$0,50 \pm 1,58 \quad 1,33 \pm 3,51 \quad 0,7682$

7. Achou difícil seguir as estratégias aconselhadas?

$1,20 \pm 2,09 \quad 0,66 \pm 2,58 \quad 0,1779$

8. Achou difícil lembrar do horário da medicação?

$2,37 \pm 3,50 \quad 0,09 \pm 0,30$

0,1178

9. Achou difícil lembrar de trocar os adesivos de nicotina?

$2,12 \pm 3,64 \quad 0,20 \pm 0,63$

0,1602

Legenda: GApp: Grupo aplicativo, CG: Grupo controle

\section{DISCUSSÃO}

Os achados do presente estudo apontam semelhança nos índices de cessação, recaída e na intensidade dos sintomas da síndrome de abstinência à nicotina de ambos os grupos analisados. Além disso, usuários do GC e GApp apresentaram níveis de satisfação semelhantes.

O índice de cessação apresentado por ambos os grupos mostraram-se elevados no período de acompanhamento do estudo, entretanto sem diferenças para o GApp que associou o tratamento presencial com utilização do aplicativo de celular. Isso mostra que a abordagem intensiva ao fumante em formato presencial associado com terapia farmacológica é um componente essencial para o sucesso na cessação.

As taxas de cessação encontradas no estudo, foram altas para ambos os grupos (GApp e GC, mesmo não havendo diferenças significativas da comparação entre eles. $\mathrm{Na}$ análise intergrupos observou-se altas taxas de cessão devido à abordagem intensiva, o que se mostra superior a aconselhamentos breves, trazendo a importância da preparação com os recursos audiovisuais, equipe interdisciplinar para aconselhamento e acompanhamento especializado ${ }^{20}$.

Whittaker e colaboradores em revisão sistemática com artigos publicados utilizando aplicativos de celular, identificaram que não Colloq Vitae 2020 set-dez; 12(3): 37-46.

Artigo Open Access sob uma licença CC BY-NC-ND (http://creativecommons.org/licenses/by-nc-nd/4.0/). houve diferença estatísticamente significante quando comparado o uso dos aplicativos com o aconselhamento mínimo de baixa intensidade, o que corrobora com nossos achados, do qual não foram identificadas essa diferença, entretanto com o diferencial de que foi realizada uma abordagem intensiva no presente estudo ${ }^{23}$.

Como já demonstrado na literatura, a abordagem intensiva tem um papel importante para a cessação tabagistica, sendo mais eficaz que a utilização do aplicativo em si, pois trata-se de uma abordagem com acompanhamento da equipe interdisciplinar, ofertando o suporte necessário aos participantes além do auxílio audiovisual ${ }^{20,23}$.

São baixas as evidências quanto ao uso de smartphones comparado com o suporte mínimo, devido a inconsistência em precisão dos aplicativos e grande variabilidade dos estudos. Isso mostra a importância de mais ensaios clínicos aleatorizados que façam essa comparação de modo a melhorar a fundamentação desse conhecimento. Apesar de saber que não há evidência efetiva, é necessário cautela visto que esses estudos possuem limitações, pois trata-se de aplicativos dispares, cujo rigor cientifico é baixo ${ }^{23}$.

Garrison e colaboradores mostraram evidências avaliando aplicativos para smartphone, porém foram criticados, pois esses aplicativos não são considerados amplamente 
acessíveis a toda população, visto que contam com um certo grau de alfabetização e familiarização digital. Vale ressaltar que nos estudos incluídos sobre aplicativos para smartphones, haviam altos níveis de escolaridade, dos quais $84 \%$ dos participantes possuiam ensino médio ao superior ${ }^{24}$.

De acordo com Haskins e colaboradores através de uma revisão sobre a disponibilidade dos aplicativos, $57 \%$ deles eram baseados em evidências de baixa qualidade e apenas $50 \%$ dos aplicativos que permanecem disponíveis para os consumidores eram baseados em conteúdos científicos. Uma análise de conteúdo recente desses aplicativos revelaram baixos níveis de adesão às diretrizes de tratamento baseadas em evidências. É necessário identificar quais aplicativos para cessação oferecem um suporte baseado em estratégias de diretrizes de tratamento e sua disponibilidade na plataforma para os consumidores de modo que 0 acompanhamento remoto tenha fundamentos para que o tratamento possa ser concluido de modo efetivo ${ }^{25}$. Formagi e colaboradores realizaram revisões do qual foram analisados aplicativos em lingua portuguesa, do qual apontaram desfechos semelhantes na falta de qualidade científica e padronização dos mesmos $^{16}$.

Os dados encontrados no presente estudo, corroboram com os achados de Baskerville e Peiris do qual não encontraram evidência significativa ao se comparar a utilização dos aplicativos para smartphone com o suporte presencial mínimo, o que demonstra baixa qualidade de evidência científica e a variação dos componentes disponíveis nesses aplicativos. Isso mostra a importância e eficácia do acompanhamento presencial intensivo para que se obtenha sucesso no tratamento ${ }^{26,27}$.

Apesar do presente estudo possuir limitações que devem ser levadas em consideração para a interpretação dos achados, sendo elas o tempo de fumo e a idade dos participantes que foram diferentes entre os grupos, espera-se que quanto maior o tempo de fumo, maior a dificuldade para a cessação, e que o fator idade pode ter sido determinante quanto ao uso do smartphone, uma vez que indivíduos com idade avançada podem apresentar menor afinidade com o uso da tecnologia. Além disso, houve um número amostral reduzido, uma vez que a taxa de desistência ao longo do acompanhamento foi alta.

Colloq Vitae 2020 set-dez; 12(3): 37-46

Artigo Open Access sob uma licença CC BY-NC-ND (http://creativecommons.org/licenses/by-nc-nd/4.0/)
Somado a este fato, a compatibilidade do aplicativo utilizado neste estudo era apenas para smartphones com sistema operacional Android ${ }^{\circledR}$ - que fez com que o recrutamento dos participantes ficasse limitado, uma vez que o aplicativo escolhido foi informativo, o qual sugere-se escolher um aplicativo com ferramentas interativas criando vínculo com os participantes como evidenciado por Bock em sua revisão sistemática ${ }^{28}$

Desse modo, pode-se concluir que o uso de um aplicativo de celular para cessação tabagística associado a tratamento presencial promoveu efeitos semelhantes ao tratamento presencial isolado no índice de cessação, síndrome de abstinência e satisfação de tabagistas.

\section{CONFLITO DE INTERESSE}

Os autores declaram não haver qualquer potencial conflito de interesse que possa interferir na imparcialidade deste trabalho científico.

\section{REFERÊNCIAS}

1. Nações Unidas 2017. Organização Mundial da Saúde (OMS). 1 em cada 5 pessoas no mundo fumam. Disponível em: https://nacoesunidas.org/oms-1-em-cada-5pessoas-no-mundo-fuma/amp/

2. Mirra AP, Meirelles RHS, Godoy I, Issa JS, Reichert J, Carvalho NB, et al. Tabagismo. Diretrizes clinicas na saúde suplementar. Associação medica brasileira e agencia nacional de saúde suplementar 2011.

3. Silva $D R$, Torrico $M M$, Duarte $R$, Galvão $T$, Bonini EH, Arbex FF, Arbex MA, Augusto VM, Rabahi MF, Mello FCQ et al. Fatores de risco para tuberculose: diabetes, tabagismo, álcool e uso de outras drogas. J bras pneumol. 2018; 44(2): 145$52 . \quad$ http://doi.org/10.1590/s1806$\underline{37562017000000443}$

4. Araújo AJ, Menezes AM, Doria AJ, Torres BS, Viegas CA, Silva CA et al. Diretrizes para cessação do tabagismo. J Bras Pneumol 2004; 30(Suppl.2): S1-S76. 37132004000800002

http://doi.org/10.1590/S1806-

5. Oliveira RM, Siqueira Junior AC, Santos JLF, Furegato ARF. Nicotine dependence in the mental disorders, relationship with clinical indicators, and the meaning for the user.Rev Latino-Am 
Enfermagem. 2014; 22(4):685-92. http://doi.org/10.1590/0104-1169.3549.2468

6. Ozgunay SF, Karasu D, Dulger S, Yilmaz C, Tabur Z. Relação entre consumo de tabaco e concentração de monóxido de carbono na expiração com complicações respiratórias perioperatórias. Rev Bras Anestesiol. 2018; 68(5): 462-71.

http:/doi.org/10.1016/j.bjane.2018.02.003

7. Cahill K, Stevens S, Perera R, Lancaster T. Pharmacological interventions for smoking cessation: an overview and network metaanalysis (Review). Cochrane Database Syst Rev. 2013.

http://doi.org/10.1002/14651858.CD009329.pub $\underline{2}$.

8. Pinheiro GP, Machado CS, Fernandes AGO, Mota RCL, Lima LL, Vasconcellos DS, et al. Tabagismo entre asmáticos: avaliação por auto relato e dosagem de cotinina urinaria. J Bras Pneumol. 2018; 44(6): 477-85. https://doi.org/10.1590/s1806-

$\underline{37562017000000018}$

9. Leventakos K, Schwecke AJ, Deering E, Cathcart-Rake E, Sanh AC, Jatoi A. The Need to Prioritize and Re-prioritize Palliative Care Options: Smoking Cessation as a Case-in-Point. Curr Treat Options in Oncol. 2019; 20(4):33-40. https://doi.org/10.1007/s11864-019-0632-7.

10. Reichert J, Araújo AJ, Gonçalves CMC, Godoy I, Chatkin JM, Sales MPU, Santos SRRA et al. Smoking cessation guidelines. J Bras Pneumol. 2008;34(10): 845-80.

http://doi.org/10.1590/S180637132008001000014

11. Anthenelli RM, Gaffney M, Benowitz NL, West $R$, McRae $T$, Russ $C$ et al. Predictors of Neuropsychiatric Adverse Events with Smoking Cessation Medications in the Randomized Controlled EAGLES Trial. J Gen Intern Med. 2019; 34(6):862-870. http://doi.org/10.1007/s11606019-04858-2.

12. Mendes ACR, Toscano CM, Barcellos RMS, Ribeiro ALP, Ritzel JB, Cunha VS et al. Custos do programa de tratamento do tabagismo no Brasil. Rev Saúde Pública 2016; 50:66. http://doi.org/10.1590/s1518$\underline{8787.2016050006303}$

13. França SA, Neves ALF, Souza TAS, Martins NCN, Carneiro SR, Sargues ESNF et al. Factors associated with smoking cessation. Rev Saúde Pública. 2015;49(10).

http://doi.org/10.1590/50034$\underline{8910.2015049004946 .}$.

14. Bertani AL, Garcia T, Tanni SE, Godoy I et al. Preventing smoking during pregnancy: the importance of maternal knowledge of the health hazards and of the treatment options available. J Bras Pneumol. 2015;41(2):175-81. DOI:http://dx.doi.org/10.1590/S1806-

$\underline{37132015000004482}$

15. Android sits atop Brazil's smartphone Market. Disponível em: http://www.emarketer.com/Article/Android-SitsAtop-Brazils-Smartphone-Market/1012573.

16. Formagini TDB, Ervilha RR, Machado NM, Andrade BABB, Gomide HP, Ronzani TM. A review of smartphone apps for smoking cessation available in Portuguese. Cad Saude Publica. 2017; 33(2):e00178215. http://doi.org/10.1590/0102$\underline{311 \times 00178215}$

17. Kong G, Elis DM, Sarin DRCSK. Text messaging-based smoking cessation intervention: A narrative review. Addict Behav. 2014;5:907917.

http://doi.org/10.1016/i.addbeh.2013.11.024

18. Brinzaniuc A, Strilciuc A, Blaga OM, Chereches $\mathrm{RM}$, Meghea $\mathrm{Cl}$. Smoking and quitting smoking during pregnancy: A qualitative exploration of the socio-cultural context for the development of a couple-based smoking cessation intervention in Romania. Tob Prev Cessation 2018;4:9. http://doi.org/10.18332/tpc/86161

19. Andrade SAS, Garcia T, Biral AT, Bertani AL, Caram LMO, Cezare TJ et al. Evaluation of smoking cessation treatment initiated during hospitalization in patients with heart disease or respiratory disease. J Bras Pneumol. 2018;44(1):42-8. http://doi.org/10.1590/s1806$\underline{37562017000000026}$

20. Freire APCF, Ramos D, Silva BSA, David RM, Pestana PRS, Fernandes RA et al. Resultados de 
um programa de cessação tabagistica: analises de novos procedimentos. ConScientiae Saúde. 2014; 13(3): 396-404. http://doi.org/10.5585/ConsSaude.v13n3.4894

21. Welsch SK, Smith SS, Wetter DW, Jorenby DE, Fiore MC, Baker TB. Development and validation of the Wisconsin Smoking Withdrawal Scale. Exp Clin Psychopharmacol. 1999; 7(4):35461. http://doi.org/10.1037/1064-1297.7.4.354

22. ORCHA 2019, Review of Quit smokingSmokerstop version 1.5.2 on Android. Diponível em https://bradfordandcraven.orcha.co.uk/review/5 276/

23. Whittaker $\mathrm{R}$, McRobbie $H$, Bullen $C$, Rodgers A, Gu Y, Dobson R. Mobile phone text messaging and app-based interventions for smoking cessation (Review). Cochrane Database Syst Rev. 2019;10(10).

https://doi.org/10.1002/14651858.CD006611.pu $\underline{\mathrm{b} 5}$

24. Garrison K, Pal P, O Malley S, Pittiman B, Gueorquieva R, Rojiani R, et al. Craving to Quit: a randomised controlled trial of smartphone appbased mindfulness trainig for smoking cessation. Nicotine Tob Res. 2020; 22(3):324-31. https://doi.org/10.1093/ntr/nty126

25. Haskins BL, Lesperance D, Gibbons MS, Boudreaux ED. A systematic review of smartphone applications for smoking cessation. Transl Behav Med. 2017; 7(2):292-9. https://doi.org/10.1007/s13142-017-0492-2

26. Peiris_D, Wright_L, News_M, Rogers_K, Redfern_J, Chow_C, et al. A smartphone app to assist smoking cessation among Aboriginal Australians: findings from a pilot randomized controlled trial. JMIR Mhealth Uhealth. 2019; 7(4):e12745. https://doi.org/10.2196/12745

27. Baskerville_N, Struik_L, Dash_D. Crush the Crave: development and formative evaluation of a smartphone app for smoking cessation. JMIR Mhealth Uhealth. 2018; 6(3):e52. https://doi.org/10.2196/mhealth.9011.

28. Bock_B, Heron_K, Jennings_E, Morrow_K, Cobb_V, Magee_J, et al. A text message delivered smoking cessation intervention: the initial trial of Colloq Vitae 2020 set-dez; 12(3): 37-46.

Artigo Open Access sob uma licença CC BY-NC-ND (http://creativecommons.org/licenses/by-nc-nd/4.0/).
TXT-2-Quit: randomized controlled trial. JMIR MHealth and UHealth. 2013; 1(2):e17. https://doi.org/10.2196/mhealth.2522 